\title{
Reconstruction tools for the study of short-lived resonances in ALICE pp collisions at the LHC startup
}

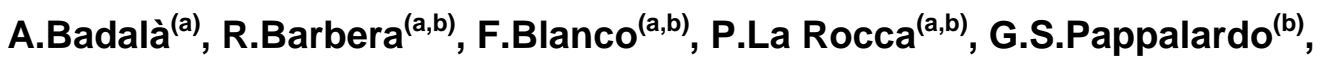 \\ A.Pulvirenti $^{(a, b)}$ F.Riggi $^{(a, b) 1}$ and R.Vernet $^{(c)}$ \\ (a) INFN, Sezione di Catania \\ (b) Department of Physics and Astronomy, University of Catania \\ (c) Consorzio Cometa, Catania \\ Via S.Sofia 64, 95123 Catania, Italy \\ E-mail: Angela.Badala@ct.infn.it,Roberto.Barbera@ct.infn.it, \\ Francesco.Blanco@ct.infn.it,Paola.Larocca@ct.infn.it, \\ Giuseppe.S.Pappalardo@ct.infn.it,Alberto.Pulvirenti@ct.infn.it, \\ Francesco.Riggi@ct.infn.it,Renaud.Vernet@ct.infn.it
}

\begin{abstract}
The study of resonances produced in proton-proton and heavy ion interactions is a powerful way of investigating the collision dynamics, since the lifetimes of many of such resonances $\left(\rho, K^{*}\right.$, $\left.\Lambda^{*}, \Phi, \ldots\right)$ are comparable to that of the hot and dense matter created in ultrarelativistic collisions. Understanding the resonance production mechanisms also in pp collisions is essential, since they provide a base reference in a supposedly purely hadronic medium. The ALICE experiment at LHC plans to carry out a study of such resonances both in pp and AA events, making use of the optimal capability of the detector to reconstruct and identify their charged decay daughters in a wide transverse momentum and rapidity range. The main observables for such a study are the resonance yields, their masses and widths, their transverse momentum spectra and particle ratios (resonances over stable particles and between different resonances). For a proper evaluation of these observables, a careful simulation study is in progress within the Collaboration, to be ready at the LHC startup. The main items of this activity include the development of analysis codes under the AliRoot environment, the evaluation of different reconstruction tools, the study of the combinatorial background, the influence of correct particle identification, the acceptance and efficiency evaluation. Most of such studies have been carried out by the use of distributed analysis within the GRID. The present status of this activity is discussed, with particular emphasis on the software tools and results expected in pp collisions at $\sqrt{s}_{\mathrm{s}}=900 \mathrm{GeV}$, when the first physics events from LHC beams could be available.
\end{abstract}

XI International Workshop on Advanced Computing and Analysis Techniques in Physics Research Amsterdam, the Netherlands

23-27 April, 2007

\footnotetext{
$1 \quad$ Speaker
} 


\section{Introduction}

The Large Hadron Collider (LHC) at CERN will produce beams of protons (up to $14 \mathrm{TeV}$ c.m. energy) and heavy ions (up to $5.5 \mathrm{~A} \mathrm{TeV} \mathrm{c.m.} \mathrm{energy).} \mathrm{The} \mathrm{ALICE} \mathrm{detector} \mathrm{[1,2]} \mathrm{is} \mathrm{one} \mathrm{of}$ the major experimental set-up at LHC, and will study both pp and AA collisions at ultrarelativistic energies. Heavy-ion collisions at such energies allow the study of a hot and dense matter, with the possibility to observe several probes giving information on the first stage of the collision. One important signal is given by the study of short-lived resonances.

In-medium modifications of meson resonances have been proposed as a possible signal of a phase transition from nuclear matter to a plasma of quarks and gluons [3]. However, even in absence of a phase transition, modifications of the properties of meson resonances can arise, due to the interaction of such particles and/or their daughters with the fireball medium $[4,5]$. Typical lifetimes of such resonances are a few fm/c, comparable to the expected lifetime of the hot and dense matter produced in such collisions.

The study of short-lived resonances may also probe the role of the rescattering phase between chemical and kinetic freeze-out. Resonance measurements are affected by two competing effects. A fraction may not be reconstructed due to the rescattering of their daughter particles. This effect depends on the time between chemical and kinetic freeze-out, the source size, the resonance phase-space distribution and the hadronic interaction cross-section of the resonances daughters. On the other side, after chemical freeze-out, pseudo-elastic interactions among hadrons in the medium may increase the resonance population. This regeneration mechanism depends on the cross-section of hadrons in the medium. Thus, the study of resonances can probe the time evolution of the source from chemical to kinetic freeze-out and test different hadronization scenarios [6].

The observation of such resonances is critical in heavy-ion experiments, because of the large background originating from the high multiplicity and from detector limitations. For a recent review of resonance measurements at SPS and RHIC energies, see [7]. Among these, the $\mathrm{K}^{*}(892)^{0}$ and $\Lambda(1520)$ have been largely investigated. The $\mathrm{K}^{*}(892)^{0}$ meson resonance and its antiparticle decay into $K \pi$, with a $\mathrm{c} \tau$ around $4 \mathrm{fm} / \mathrm{c}$, while the $\Lambda(1520)$ resonance decays into $\mathrm{pK}$, with a $\mathrm{c} \tau$ around $13 \mathrm{fm} / \mathrm{c}$ [8]. Their decay products may therefore be considered as originating from the primary vertex of the collision as far as the tracking is concerned. Reconstruction of such resonances for $\mathrm{Pb}-\mathrm{Pb}$ collision at $\mathrm{LHC}$ energies will be challenging due to the expected high multiplicity environment. Here a simulation study of various resonances in pp collisions at $900 \mathrm{GeV}$ and at $14 \mathrm{TeV}$ is discussed. Some results on this topic were already published in [9-11].

\section{The ALICE experiment}

ALICE is the LHC experiment dedicated to heavy-ion physics [1,2]. The ALICE detector includes a central part (spanning the central rapidity region $-0.9<\eta<0.9$ ), inside the L3 magnet, which provides a weak solenoidal magnetic field $(0.2-0.5 \mathrm{~T})$, and a forward part (covering the large rapidity region). The central part has several detectors for tracking and 
particle identification: the ITS (Inner Tracking System), the TPC (Time Projection Chamber), the TRD (Transition Radiation Detector) and the TOF (Time-Of-Flight). While all these detectors have a complete coverage in azimuth, the central region has also two additional detectors with partial azimuth coverage: the HMPID (High Momentum Particle Identification Detector) and the PHOS (Photon Spectrometer). An additional electromagnetic calorimeter (EMCal) will soon be included in the ALICE design. In the forward rapidity region $(-4<\eta<-$ 2.5) the Muon Spectrometer will detect muon pairs. Additional detectors are the ZDC (Zero Degree Calorimeter), the PMD (Photon Multiplicity Detector), the FMD (Forward Multiplicity Detector) and the V0 and T0 detectors, for more specialized tasks. A layout of the ALICE detector is shown in Fig.1.

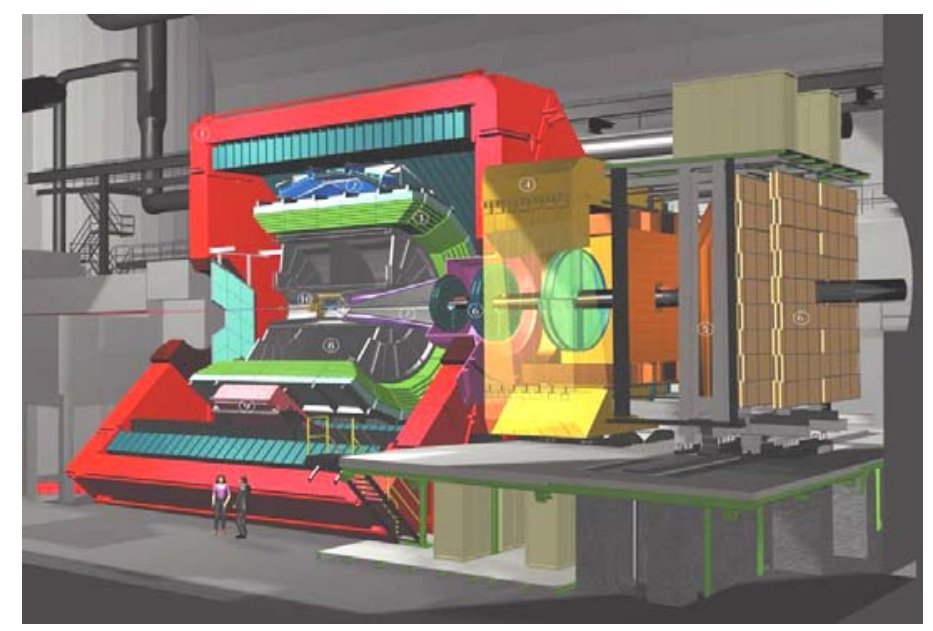

Fig.1: Layout of the ALICE detector.

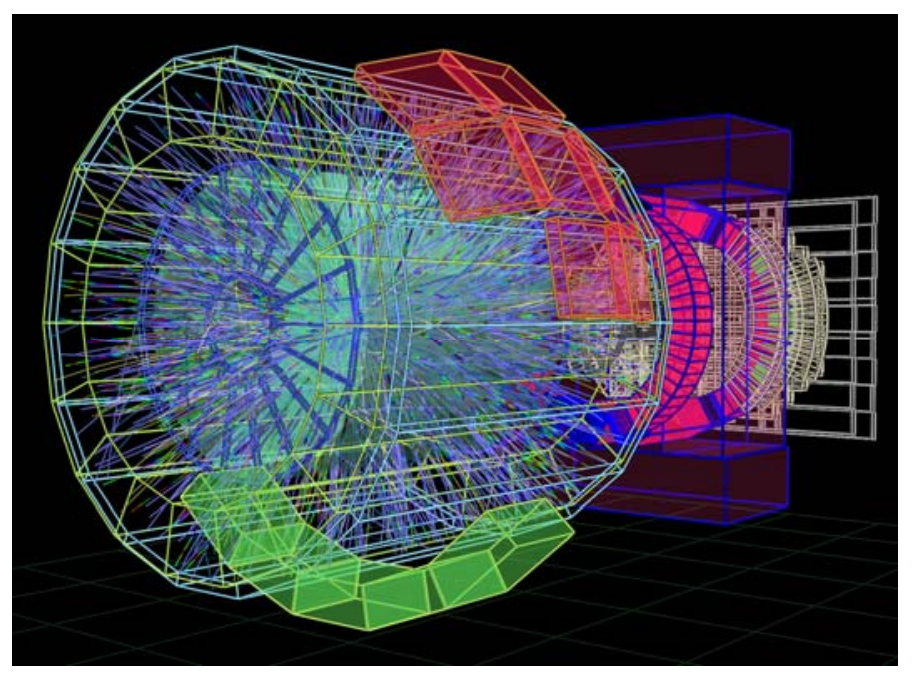

Fig.2: Charged tracks with $\mathrm{p}_{\mathrm{T}}<1 \mathrm{GeV} / \mathrm{c}$ in the ALICE barrel. 
ALICE will track and identify products from $\mathrm{pp}$ and $\mathrm{PbPb}$ collisions in a large multiplicity environment. As an example, fig.2 shows a set of charged tracks in the central region of the detector, for $\mathrm{p}_{\mathrm{T}}<1 \mathrm{GeV} / \mathrm{c}$.

\section{Short-lived resonances in ALICE}

Table I reports a list of the short-lived resonances of interest for the ALICE experiment, together with their lifetimes.

\begin{tabular}{|ll}
\hline Resonance & Life-time $[\mathrm{fm} / \mathrm{c}]$ \\
$\rho$ & 1.3 \\
$\Delta^{++}$ & 1.7 \\
$\mathbf{f}_{\mathbf{0}}(\mathbf{9 8 0})$ & 2.6 \\
$\mathbf{K}^{*}(\mathbf{8 9 2})$ & 4.0 \\
$\Sigma(1385)$ & 5.7 \\
$\Lambda(\mathbf{1 5 2 0})$ & 13 \\
$\omega(\mathbf{7 8 3})$ & 23 \\
$\Phi(\mathbf{1 0 2 0})$ & 45
\end{tabular}

Table I: List of short-lived resonances.

The main observables concerning the study of short-lived resonances are: their yields, the measure of their masses and widths, their transverse mass and transverse momentum spectra, and the particle ratios. Recently, elliptic flow and the nuclear modification factors have also been addressed in case of short-lived resonances. Table II shows a (partial) list of shortlived resonances which are presently under investigation in our group.

\begin{tabular}{|ccclc|}
\hline Resonance & $\mathrm{K}^{*}(892)$ & $\Phi(1020)$ & $\Lambda^{*}(1520)$ & $\Delta(1232)$ \\
& & & & \\
Decay channel (B.R.) & $\mathrm{K} \pi(\sim 100 \%)$ & $\mathrm{K}^{+} \mathrm{K}^{-}(49 \%)$ & $\mathrm{N} \mathrm{K}(45 \%)$ & $\mathrm{N} \pi(100 \%)$ \\
Width [MeV/c $\left.\mathrm{c}^{2}\right]$ & 50.8 & 4.5 & 15.6 & 100 \\
Life time $[\mathrm{fm} / \mathrm{c}]$ & 3.9 & 44 & 13 & 1.7 \\
& & & & \\
\hline
\end{tabular}

Table II: List of short-lived resonances presently under investigation in ALICE.

The present scenario for the startup of the LHC beams plans two low energy colliding proton beams for a short period, of the order of a few days. At the envisaged energy $(900 \mathrm{GeV})$ 
the number of expected events could be in the order of $10^{5}-10^{6}$. To understand to what extent is the study of short-lived resonances, at least those which have a larger yield, feasible in such conditions, we carried out a simulation study on a sample of $2 \times 10^{5}$ minimum bias PYTHIA pp events, analyzed on a local PC farm via LSF (Load Sharing Facility). An additional study was undertaken at the full LHC energy, $14 \mathrm{TeV}$, on a sample of $1.5 \times 10^{6}$ events, using GRID facilities for the analysis. Particular emphasis was given in the study to the problem of particle identification and its influence on the resonance performance, and to the evaluation of the combinatorial background. A detailed set of results is here reported only for the $\mathrm{K}^{*}(892)^{0}$ resonance, while similar results were obtained for the other resonances under study.

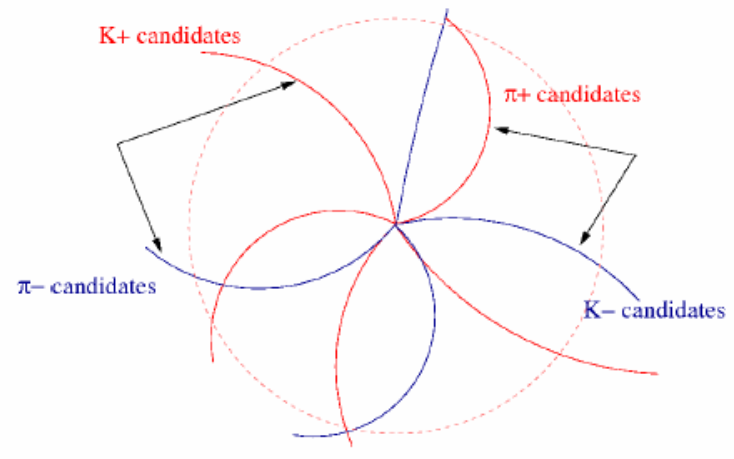

Fig.3: Unlike-sign pairs of $\mathrm{K}$ and $\pi$ candidates.

The reconstruction of short-lived resonances requires optimal performance on different aspects:

\section{Primary and secondary vertex reconstruction}

The primary and secondary vertices are mainly reconstructed in ALICE by means of the two innermost pixel layers, with resolutions as good as a few microns in case of $\mathrm{PbPb}$ collisions, and up to 40 microns in case of pp collisions. The algorithms employed take into account the 3D nature of the vertex, whose position may change in different fillings of the LHC beam.

\section{Tracking efficiency}

Tracking strategies in ALICE make use of the Kalman filter method through the ITS and TPC detectors. Such strategy allows a good tracking performance, down to very low momenta. Methods based on neural network algorithms have also been implemented for stand-alone tracking in the ITS.

\section{Momentum resolution}

Concerning the momentum resolution, the inclusion of ITS, TPC and TRD detectors results in a $\mathrm{p}_{\mathrm{T}}$ resolution as good as $3 \%$ up to $100 \mathrm{GeV} / \mathrm{c}$, and correspondingly smaller for momenta of the order of $1 \mathrm{GeV} / \mathrm{c}$, which are relevant for the bulk of the resonance yields. 


\section{Track impact parameter resolution}

The information on impact parameter is mainly provided by the ITS. The resolution on the transverse impact parameter is smaller than 100 microns in the $\mathrm{GeV} / \mathrm{c}$ region.

\section{Particle identification}

Charged particle identification is achieved at low momenta, from $0.2 \mathrm{GeV} / \mathrm{c}$, by means of a combined $\mathrm{dE} / \mathrm{dX}$ information on the silicon layers of the ITS and on the TPC. At higher momenta, in the order of a few $\mathrm{GeV} / \mathrm{c}$, the TOF detector is able to provide an optimal identification. A Bayesian approach is often used to combine the PID information originating from different detectors.

Referring, as an example, to the case of $\mathrm{K}^{*}(892)^{0}$ resonance, for the signal extraction, correlations inside each event between unlike sign pairs of $\mathrm{K}$ and $\pi$ candidates are searched for, to evaluate the invariant mass spectrum.

The combinatorial background has been studied by two different approaches: the mixedevent technique (Fig.4) and the like-sign technique (Fig.5).

In the first approach the two particles are correlated from different events, with some restriction on the similarity of the events themselves, while in the like-sign technique kaons and pions with the same charge are combined together to build the invariant mass spectrum.

The framework for the generation and reconstruction of the physics events in ALICE is based on AliROOT (Fig.6).

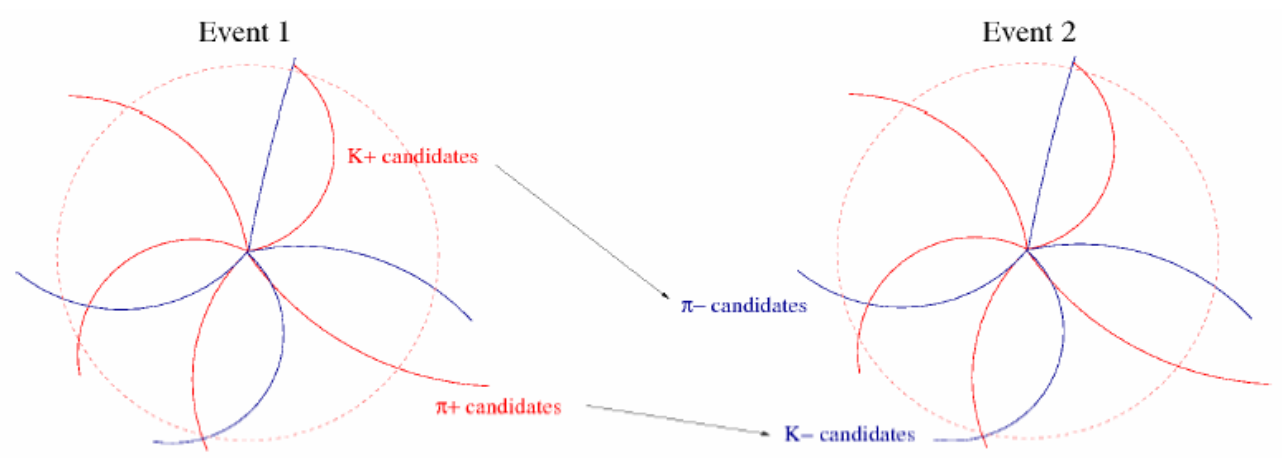

Fig.4: Mixed event technique for the evaluation of the combinatorial background. 


\section{Results from local and distributed analysis}

\subsection{Local analysis of $900 \mathrm{GeV}$ pp events}

A local analysis of 200,000 minimum bias pp events was performed via LSF on a local cluster of 60 multiprocessor CPU, with up to 30 simultaneous jobs running. In such context, one job included $100 \mathrm{pp}$ events, and the CPU time/event was in the order of $110 \mathrm{~s}$. The total CPU time spent for such analysis was about 200 days, corresponding to 10 effective days. The procedure starts from generation and reconstruction, which produces the ESD (Event Summary Data) files, together with the information on kinematics. From the ESD, a selection of primary and identified tracks produces the AOD (Analysis Object Data), with a reduction size factor of approximately 200 . From there on, one can apply kinematical cuts, further selections, draw histograms,... .

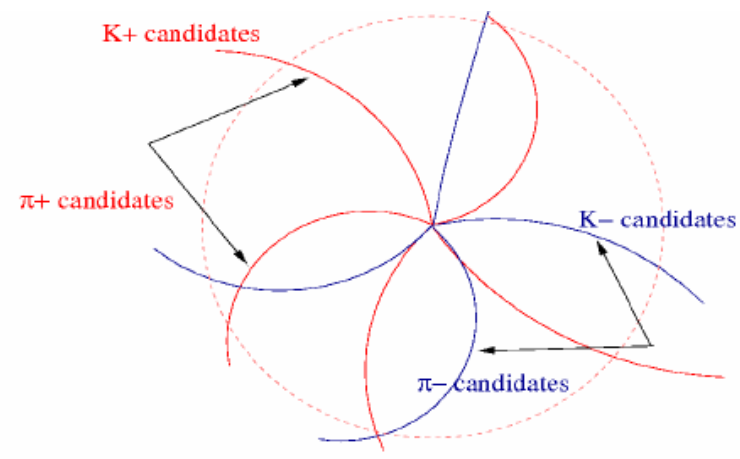

Fig.5: Like-sign technique for the evaluation of the combinatorial background.

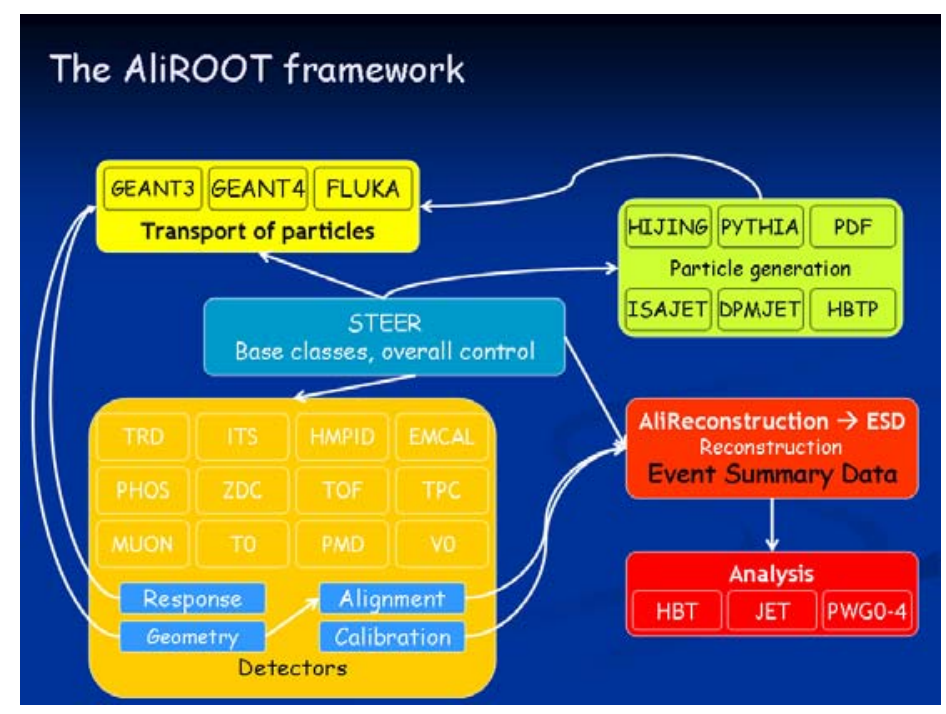

Fig.6: The AliROOT framework for the generation and reconstruction of physics events in ALICE. 
First of all, the effect of event selection on the event mixing procedure was studied. Such effect was evaluated by looking at the ratio between the True Background (estimated from the difference between Signal and True Pairs) and the Mixed Event Background.

When no selection is applied to the pair of events, this ratio is sensibly different from the unity at higher invariant masses (Fig.7), whereas a selection of the events made on the multiplicity difference $(\Delta \mathrm{m}<5)$ and on the location of the primary vertex $(\Delta \mathrm{z}<3 \mathrm{~cm})$, results in a constant ratio close to one (Fig.8). It was also verified that the selection in multiplicity is more effective than that in the primary vertex location.

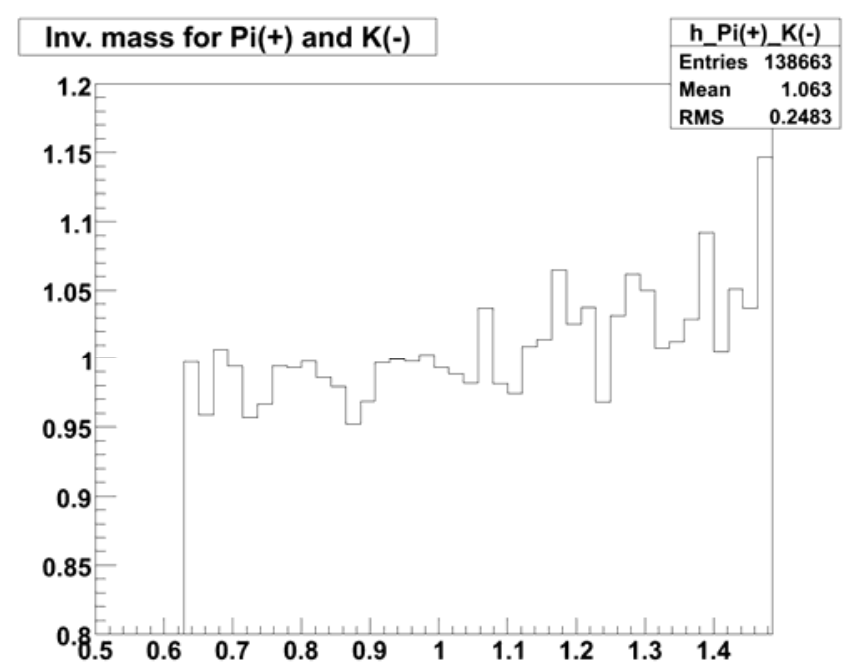

Fig.7: Ratio between the True Background and the Mixed Event Background, when no selection is applied to the event pairs.

The use of the like-sign technique was also seen to give good results in such a case. Fig.9 shows the overall results concerning the $\mathrm{K}^{*}(892) 0$ resonance. The upper left plot shows the comparison between the signal spectrum and the combinatorial one. The difference between the two, which allows to extract the signal, is shown in the upper right plot, together with the true pairs. The two lower plots give an indication of the goodness of the combinatorial background. The lower left plot compares the True Background with the Like-Sign Background, whose ratio is plotted in the lower right plot.

Concerning the problem of particle identification (PID), the information given by the TPC and TOF was used in the present analysis, with a Bayesian procedure to evaluate the overall PID probabilities. Different scenarios were considered: the perfect PID capability, and various possibilities for the realistic PID.

Fig.10 shows the mass invariant spectrum in the case of perfect PID, compared to the combinatorial background, evaluated by the event mixing technique. Especially the kaon identification may be critical, so that in a different scenario, the performance of identification 
for such particles was improved in the Bayesian algorithm by introducing a threshold on the maximal probability. Figs.11 and 12 shows the identification efficiency for kaons, without and with such improvement. The best performance shown in Fig. 12 is of course obtained at the expense of the absolute yield.

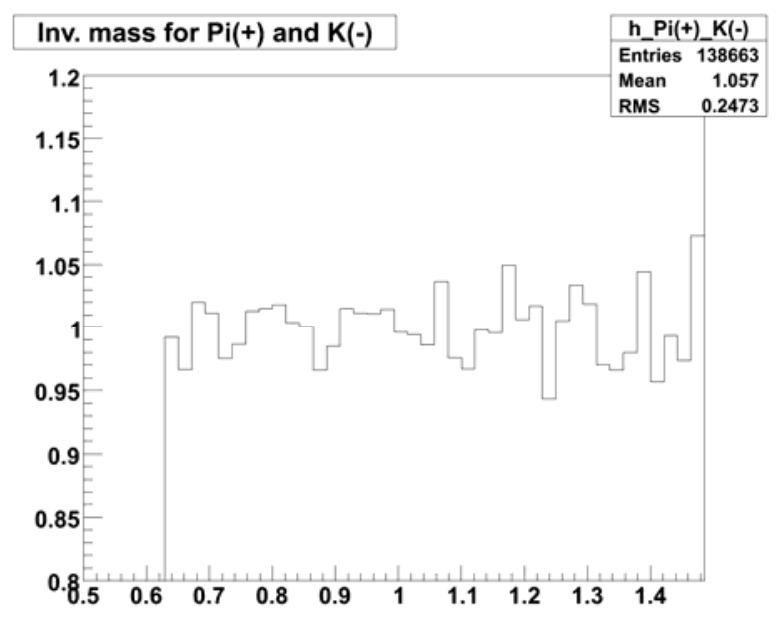

Fig.8: Ratio between the True Background and the Mixed Event Background, with selections on the event pairs.

With this choice, the invariant mass spectrum shown in Fig. 13 is obtained. A summary of the PID performance is shown in Fig.14 according to the different possible choices.

Similar analyses were also done on other resonances. Figs.15 and 16 show two of such examples, for the $\Phi(1020)$ and the $\Lambda(1520)$ respectively.

As a consequence of such analysis, we could estimate in a sample of 200,000 events, the yields and particle ratios uncertainties in the observed resonances. Table III shows the results.

\subsection{GRID distributed analysis of $14 \mathrm{TeV}$ pp events}

More sophisticated analyses of the simulated events (i.e. transverse momentum analysis, mass shifts,...) require a huge number of events and powerful computing resources. For such reason, a distributed analysis of $1.5 \mathrm{M}$ pp events at $14 \mathrm{TeV}$ from the PDC06 mass production was carried out on the GRID via AliEn (ALICE Grid Environment). This makes use of the different sites involved in the production, storage and analysis of ALICE events (Fig.17).

A typical result of such analysis is shown in Fig.18, which reports the invariant mass spectrum of kaon-pion pairs, compared with the combinatorial background estimated according to the like-sign pair method. The background subtracted signal is shown in Fig.19, together with 
the parameters (mass and width extracted from the peak). An additional analysis as a function of the transverse momentum was made possible by the high statistics achieved in the present work. Fig. 20 shows some results, for transverse momenta from zero to $4 \mathrm{GeV} / \mathrm{c}$.

Finally, a correction matrix was extracted as a function of the pseudorapidity and transverse momentum, which can be used to correct real data once measured. A lego plot of this matrix is shown in Fig.21.
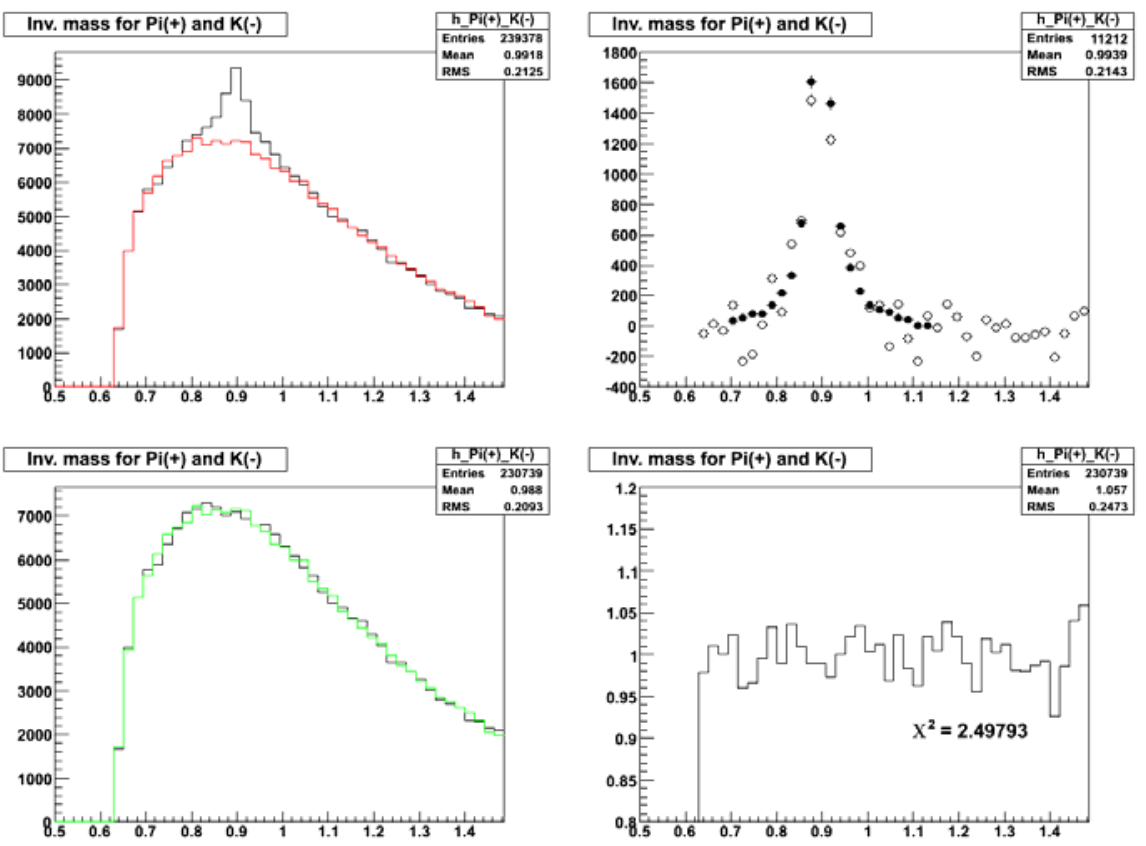

Fig.9: Results on the $\mathrm{K}^{*}(892)^{0}$ resonance with the like-sign technique.

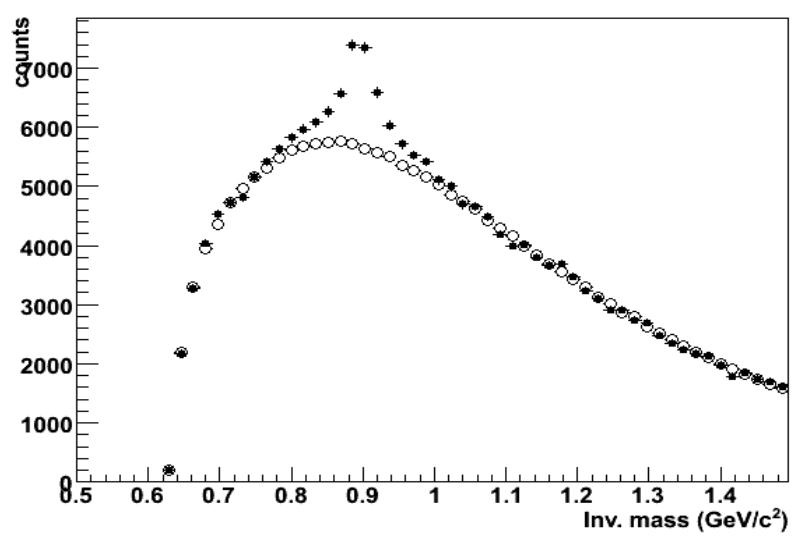

Fig.10: Invariant mass spectrum of kaon-pion pairs, with perfect PID. 


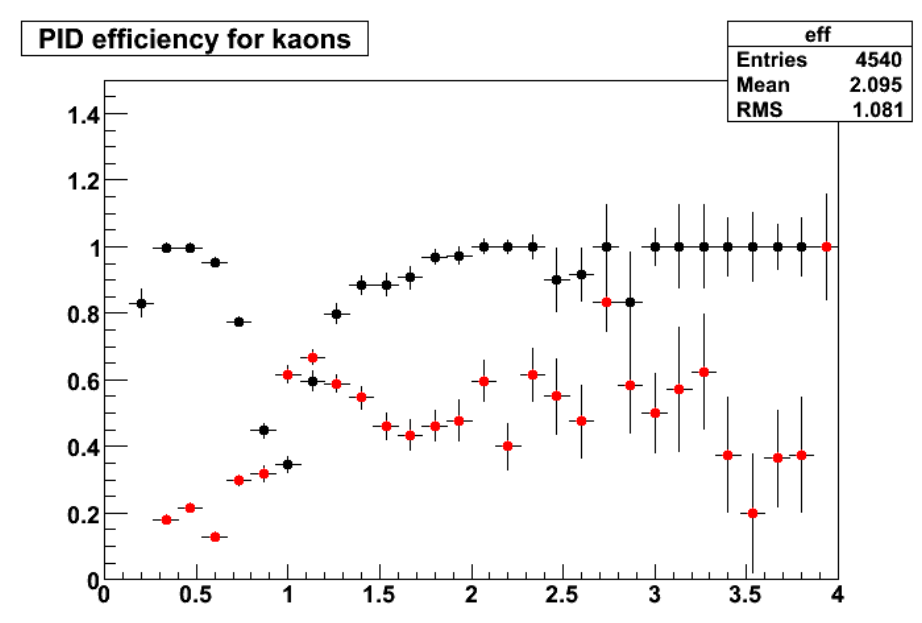

Fig.11: PID efficiency for kaons.

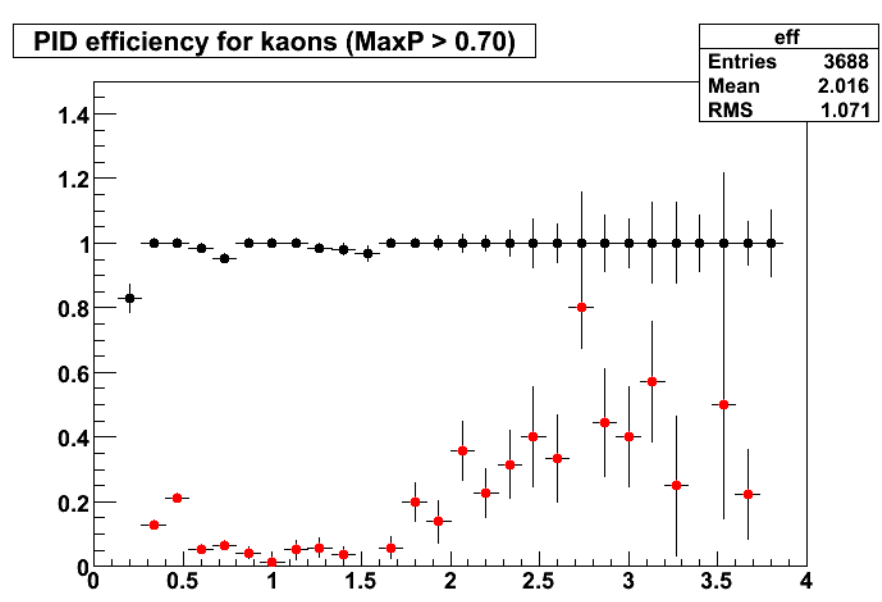

Fig.12: Improved PID efficiency for kaons. 


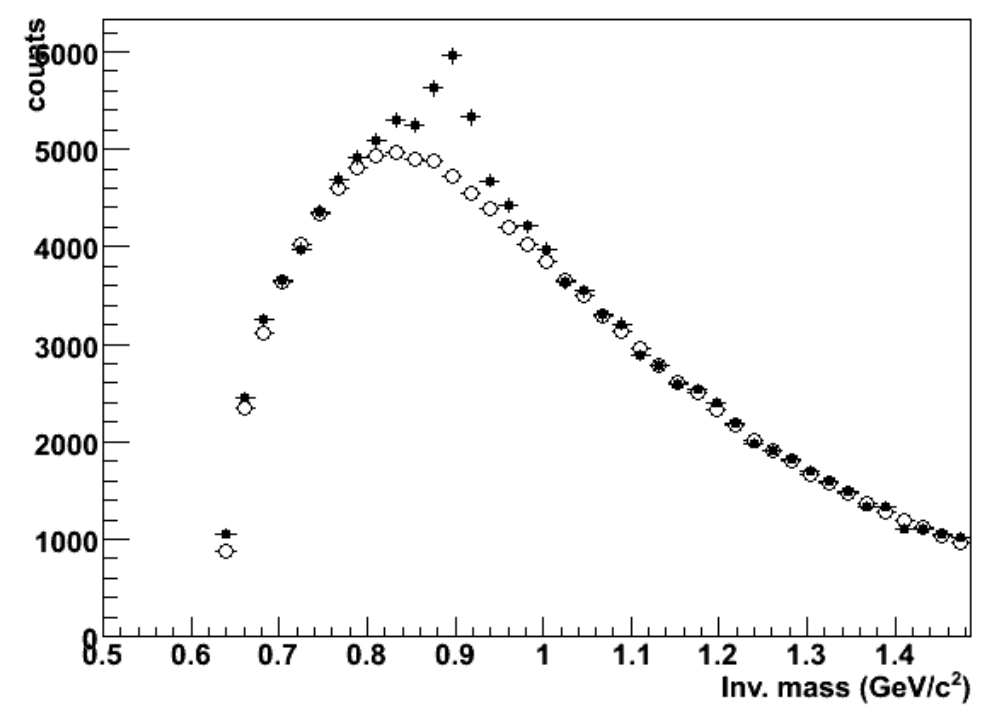

Fig.13: Invariant mass spectrum, with improved PID on kaons.

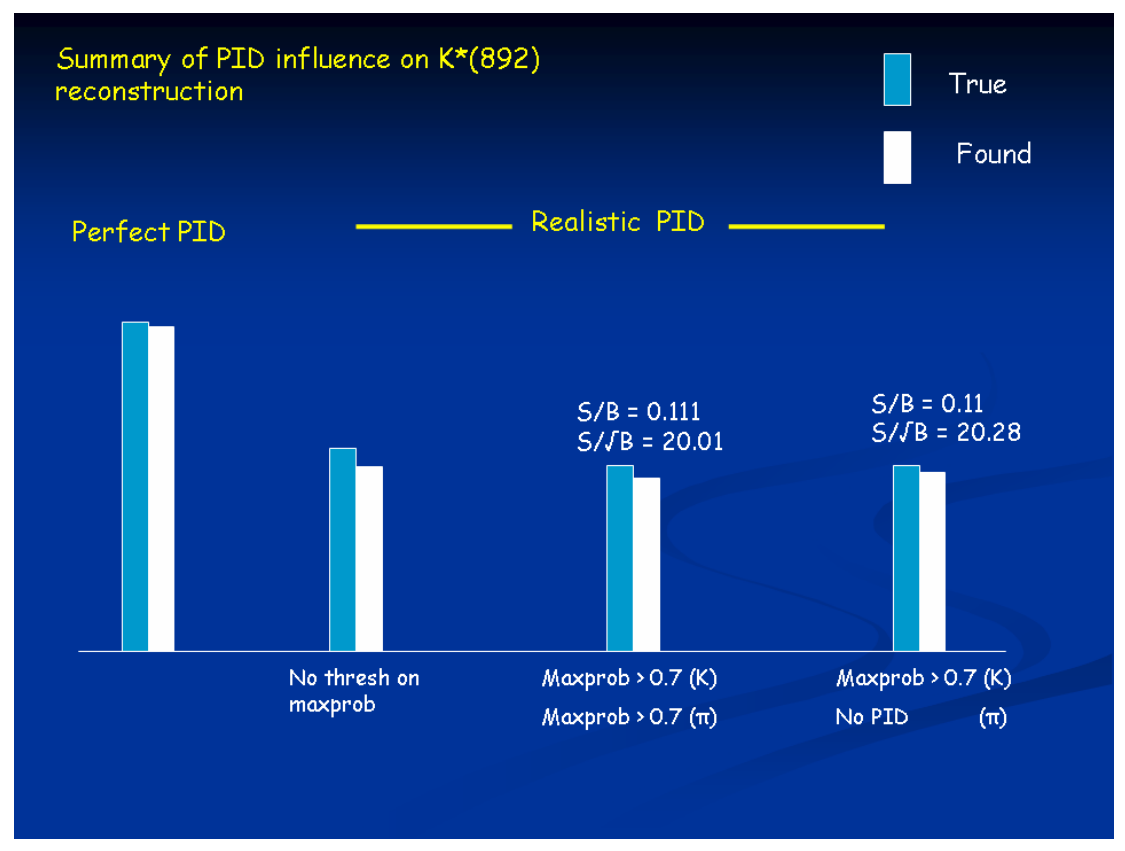

Fig.14: Summary of PID performance in the reconstruction. of the $\mathrm{K}^{*}(892)^{0}$ resonance. 


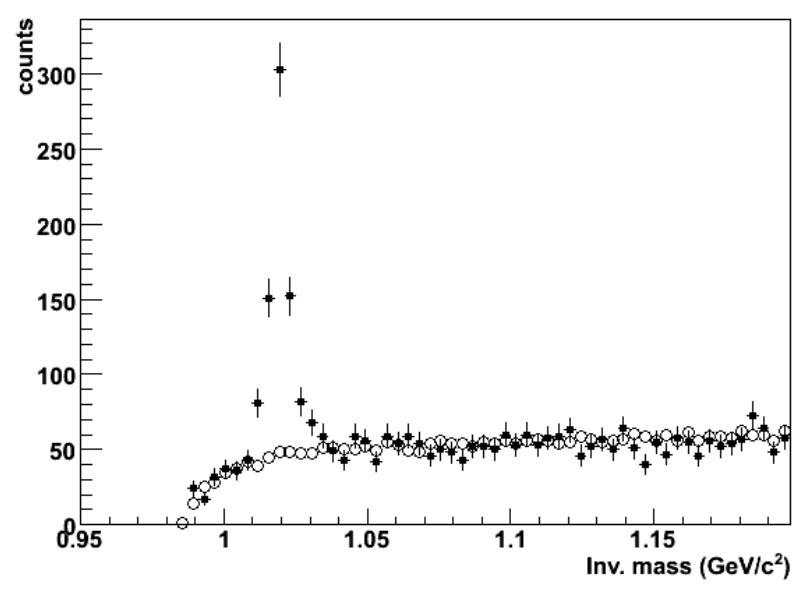

Fig.15: Invariant mass spectrum for the $\Phi(1020)$.

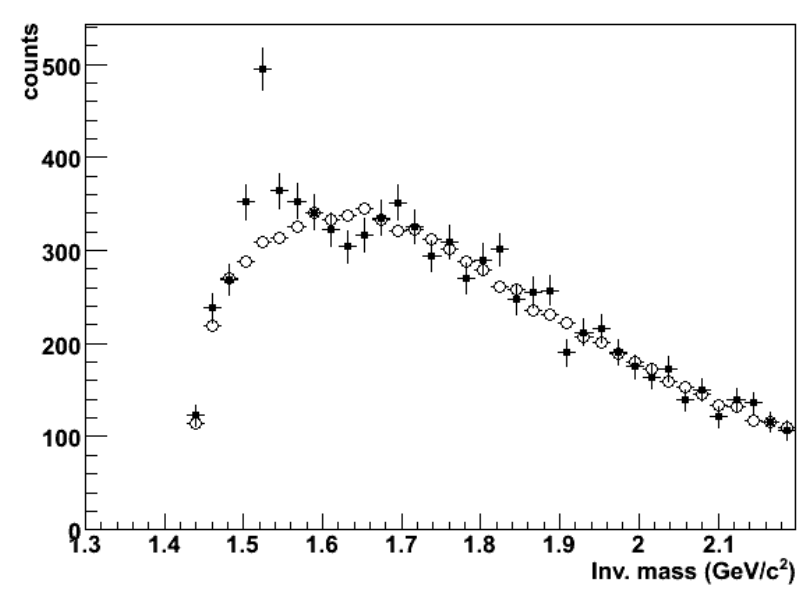

Fig.16: Invariant mass spectrum for the $\Lambda(1520)$. 


\begin{tabular}{|lc|}
\hline Particle ratios & Statistical \\
$K^{\star} / K^{-}$ & $1.6 \%$ \\
$\Lambda^{\star} / \Lambda$ & $9 \%$ \\
$\Phi / K^{\star}$ & $8 \%$ \\
$\Phi / \Lambda^{\star}$ & $8 \% \%$ \\
$K^{\star} / \Lambda^{*}$ & $12 \%$ \\
\hline
\end{tabular}

Table III: Statistical uncertainties on the resonance particle ratios.

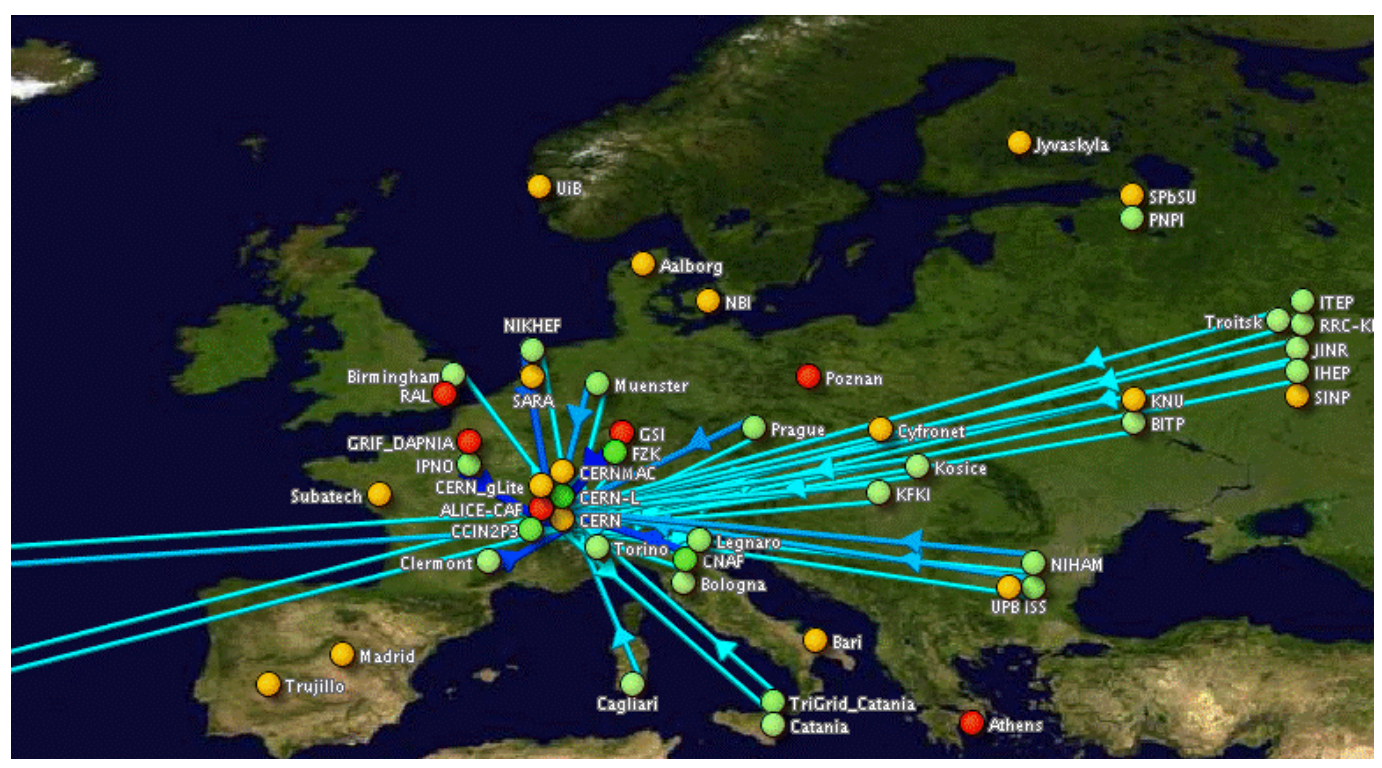

Fig. 17: The links of the different sites involved in the ALICE production and analysis. 


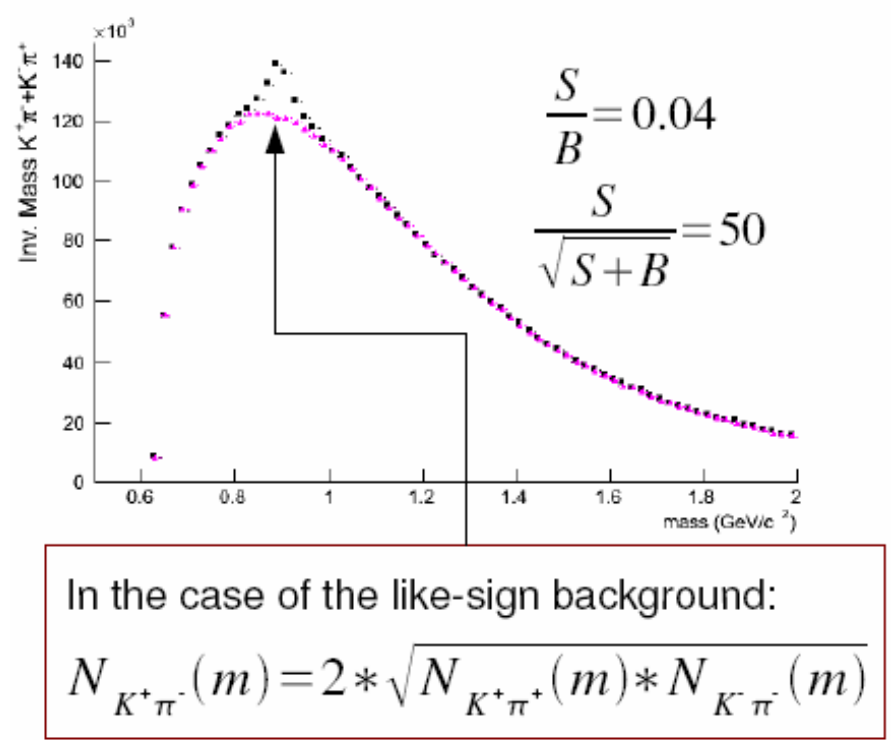

Fig.18: Invariant mass spectrum of kaon-pion pairs, from the analysis of 1.5 Mevents.

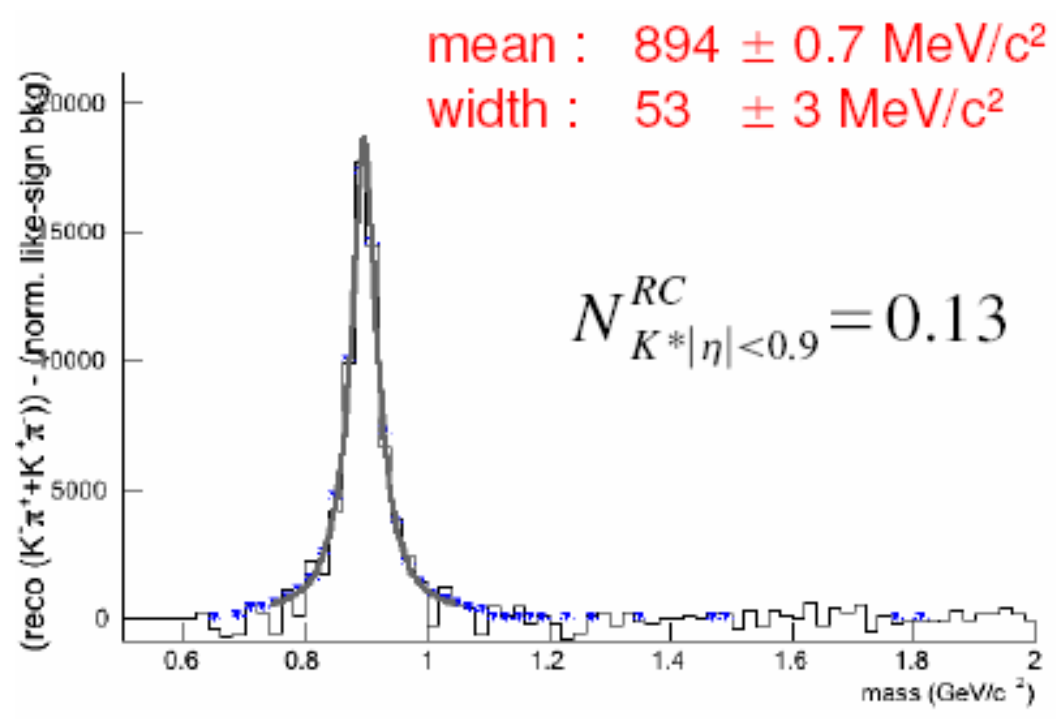

Fig.19: Invariant mass spectrum of kaon-pion pairs, after subtraction of combinatorial background. 


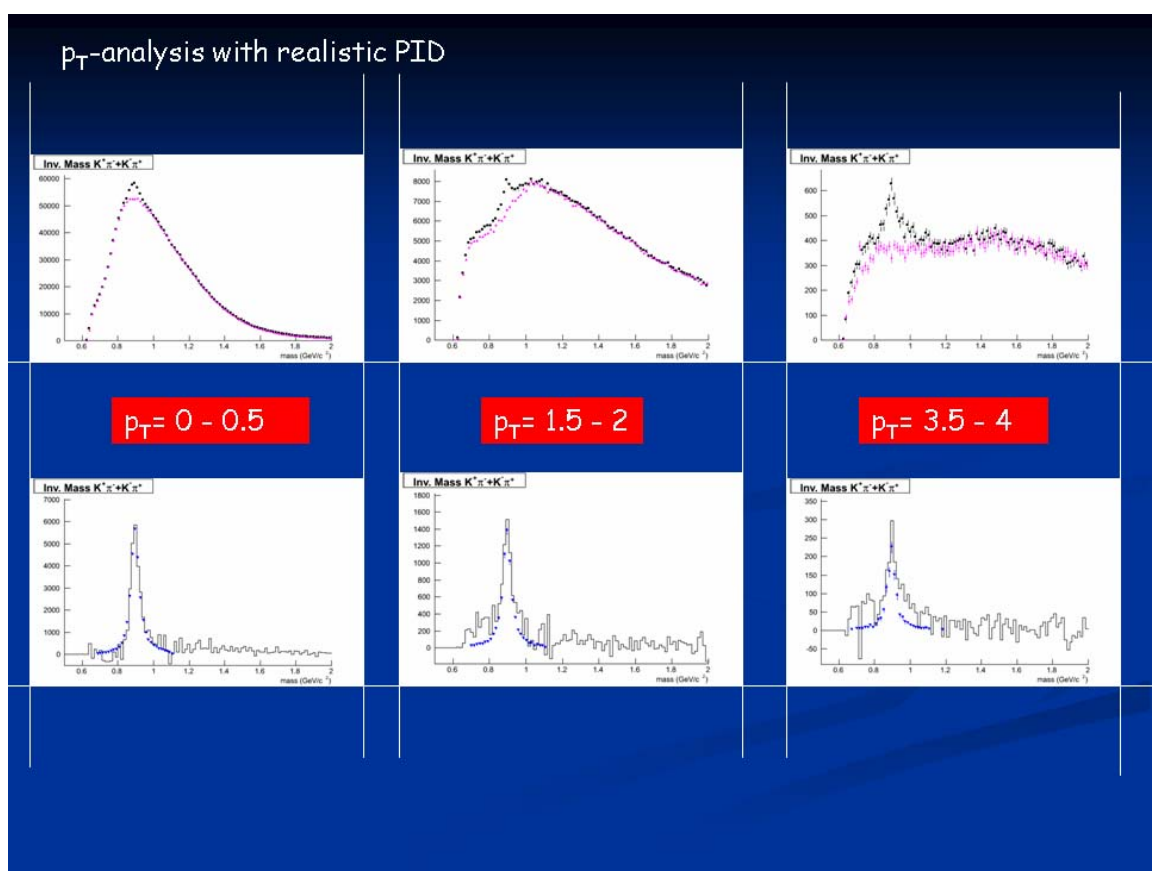

Fig.20: Invariant mass spectra, for different bins of the transverse momentum (in $\mathrm{GeV} / \mathrm{c}$ ).

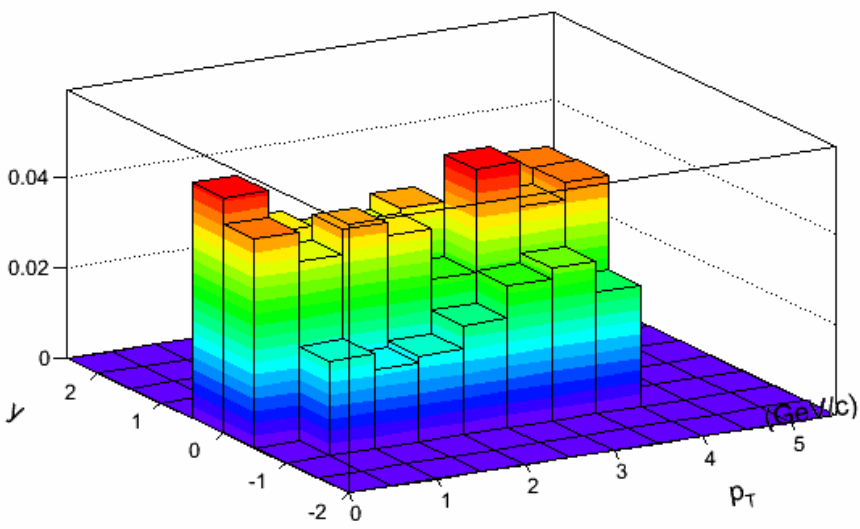

Fig.21: The correction matrix for the $\mathrm{K}^{*}(892)^{0}$ resonance is plotted here as a function of the rapidity and transverse momentum. 


\section{Conclusions}

The present study of short-lived resonances in $\mathrm{pp}$ and $\mathrm{PbPb}$ collisions at LHC energies demonstrates that such probes can be investigated with ALICE from the very beginning.

With a small sample of events, $\mathrm{O}\left(10^{5}\right)$, and realistic PID it should be possible to extract the yields at least for $\mathrm{K}^{*}(892), \Phi(1020), \Lambda^{*}(1520)$, and to measure a rough $\mathrm{p}_{\mathrm{T}}$ - distribution for $\mathrm{K}^{*}(892)$ up to $1.5-2 \mathrm{GeV} / \mathrm{c}$ and the particle ratios $\Phi / \mathrm{K}^{*}, \Lambda^{*} / \mathrm{K}^{*}, \Phi / \Lambda^{*}$ with uncertainties in the order of $10 \%$.

The analysis of a larger sample, $\mathrm{O}\left(10^{6}\right) \mathrm{pp}$ events at $14 \mathrm{TeV}$ fully reconstructed on the GRID has allowed to conclude that in such a case the resonance yields are obtainable with large statistics. Moreover, a complete $\mathrm{p}_{\mathrm{T}}$-analysis may be performed. Using such sample of events also permitted to evaluate the correction matrix $\left(\mathrm{y}, \mathrm{p}_{\mathrm{T}}\right)$.

Additional studies in progress will focus the extension of such approach to other resonances as well.

\section{References}

[1] ALICE Physics Performance Report Vol. I, J. Phys. G30 (2004)1517

[2] ALICE Physics Performance Report Vol. II, J. Phys. G32 (2006 )1295

[3] R. Rapp and J. Wambach, Adv. Nucl. Phys. 25 (2000) 1

[4] E.V. Shuryak and G.E. Brown, Nucl. Phys. A717 (2003)322.

[5] R. Rapp, Nucl. Phys. A725 (2003)254.

[6] J. Letessier et al. , J. Phys. G27 (2001)427

[7] C. Markert, J. Phys. G31 (2005)S196.

[8] Particle Data Group, K. Hagiwara et al. , Phys. Rev. D66 (2002)010001.

[9] A. Badalà et al., ALICE Internal Note ALICE/INT 2003-31.

[10] A. Badalà et al., Nucl. Instr. and Meth. in Phys. Res. A534 (2004)189.

[11 A. Badalà et al., ALICE Internal Note ALICE/INT 2005-39. 